\title{
Informativity of transient processes accompanying hydrodynamic well survey
}

\author{
Shakirov Albert Amirzyanovich \\ Ufa State Petroleum Technological University, \\ Branch of the University in the City of Oktyabrsky, Russian Federation \\ albert551@yandex.ru
}

\begin{abstract}
Annotation - One of special GWL methods included into mandatory complexes of studies in open holes is the formation testing with logging cable also known as hydrodynamic well survey. Over 60 years, Russian company VNIIGIS have been developing the equipment, method and technology for formation testing with logging cable. At the stages of exploration and survey, hydrodynamic well logging allows determining the coverage of the reservoir, its permeability and fluid properties. During the development, the major attention is drawn by the problems of estimation of reservoir pressure, flow rate and compartmentalization. At later stages of oil field development, the researchers need to choose the methods of secondary exploitation, which requires determining the reservoir behavior. To achieve these goals at each of the stages of oil field development, one should measure the downhole reservoir pressure, temperature, flow rate during pay testing; Representative samples of reservoir fluid should be picked. Technical characteristics of AGIP-K hardware complex allow reaching and performing the afore mentioned goals and tasks. The work presents the analysis of pressure build-up curves during hydrodynamic survey of hydrocarbon wells. The data received from oil fields of Tatarstan, Western Siberia and Yakutia are considered. The results of the study of transient processes during hydrodynamic survey allows establishing the distribution of parameters that characterize filtration and elastic properties of the rocks and fluids of hydrocarbon and water sites.
\end{abstract}

Keywords - hydrodynamic survey, statistical dependence, measuring chamber, sample picker, amplitude-time characteristic, transient process, filtration and elastic properties of rocks.

\section{INTRODUCTION}

The technology of hydrodynamic survey implemented in the reservoir tester with AGIP-K cable provides isolation of a part of reservoir from a well, adjustable pressure decrease below reservoir pressure, filling of measurement chambers at required pressure difference, transportation of the reservoir fluid to the surface for determination of reservoir saturation character, measurement of inflow pressure and build-up pressure [1-14].

The geological problems covered by AGIP-K hardware and methodological complex include:

detection of inflows from potential pay zones (according to data from GWL); reservoir

selection of permeable zones inside nonuniform

determination of effective thickness of pays;

determination of reservoir pressure across the section to generate or refine the hydrodynamic model of the oil field;

- determination of the fluid mobility ratio in reservoirs; establishment of the productivity profile and estimation of potential flow rates of the reservoirs;

refinement of the location of interfluid contacts in the section;

determination of the character of reservoir saturation.

Present work analyses transient processes during hydrodynamic survey. The curves for the analysis were received from oil fields of Tatarstan, Western Siberia and Yakutia.

\section{MATERIALS AND METHODS}

Reservoir testing (hydrodynamic survey) was performed in line with "Technical instruction for geophysical study and operation of equipment with cable in oil and gas wells" (RD 153-39.0-072-01).

\section{METHOD CONTENT AND ESTIMATION OF ITS EFFICIENCY}

The determination of filtration and elastic characteristics of hydrodynamically surveyed reservoir zones requires objective and differentiated consideration of many factors accompanying the process of hydrodynamic disturbance and affecting the character of recorded pressure diagrams. The stage especially sensitive to factors in effect is so-called closed period of transient process during elastic redistribution of pressure during inflow and after stoppage of the differential piston of the sampler measurement chamber. Among the factors affecting the pressure curve, the most specific are the difference in physical properties of the fluids in the reservoirs: oil, gas, water and their mixture.

The discrepancies in the physical properties of oil, gas and reservoir water can affect the pressure diagrams by contributing a specific character to their certain phases, especially at the transient process stage.

Fig. 1 depicts an element of the pressure diagram which characterizes the effect of factors conditioned by the discrepancy in the properties of fluids on the curve configuration in the transient process. Characteristic are points $O$ and $B$, the points of transient process beginning and end, 
which duration equals $t_{B} . A_{1}, A_{2}, A_{3}$ are points where curve starts leaving the slope with maximum steepness. $D_{1}, D_{2}, D_{3}$ are points where curve ends entering the slope with maximum steepness, which duration is determined by $t_{A 1}, t_{A 2}, t_{A 3}$.

The statistical dependence of transient process time $t_{B}$ and time of fluid inflow into the third stage of the sampler or measuring chamber of the differential piston of the inflow void $t_{i}$. The values of $t_{B}$ and $t_{i}$ were classified according to the medium (water, oil and gas). Since the range of time change turned out to be significant for $t_{i}$ from 0.1 to 1000 seconds and $t_{B}$ from 0.1 to 100 seconds, the dependence $t(t)$ was plotted in logarithmic coordinates. In the array of values of $t_{B}\left(t_{i}\right)$, there is a possibility of occurrence of values disturbed by hardware or technological factors connected with leakage or clogging of valves due to various additives in the flush liquid. Unfortunately, the latter plays a significant role in the estimation of the saturation character of tested objects using hydrodynamic survey pressure diagrams.

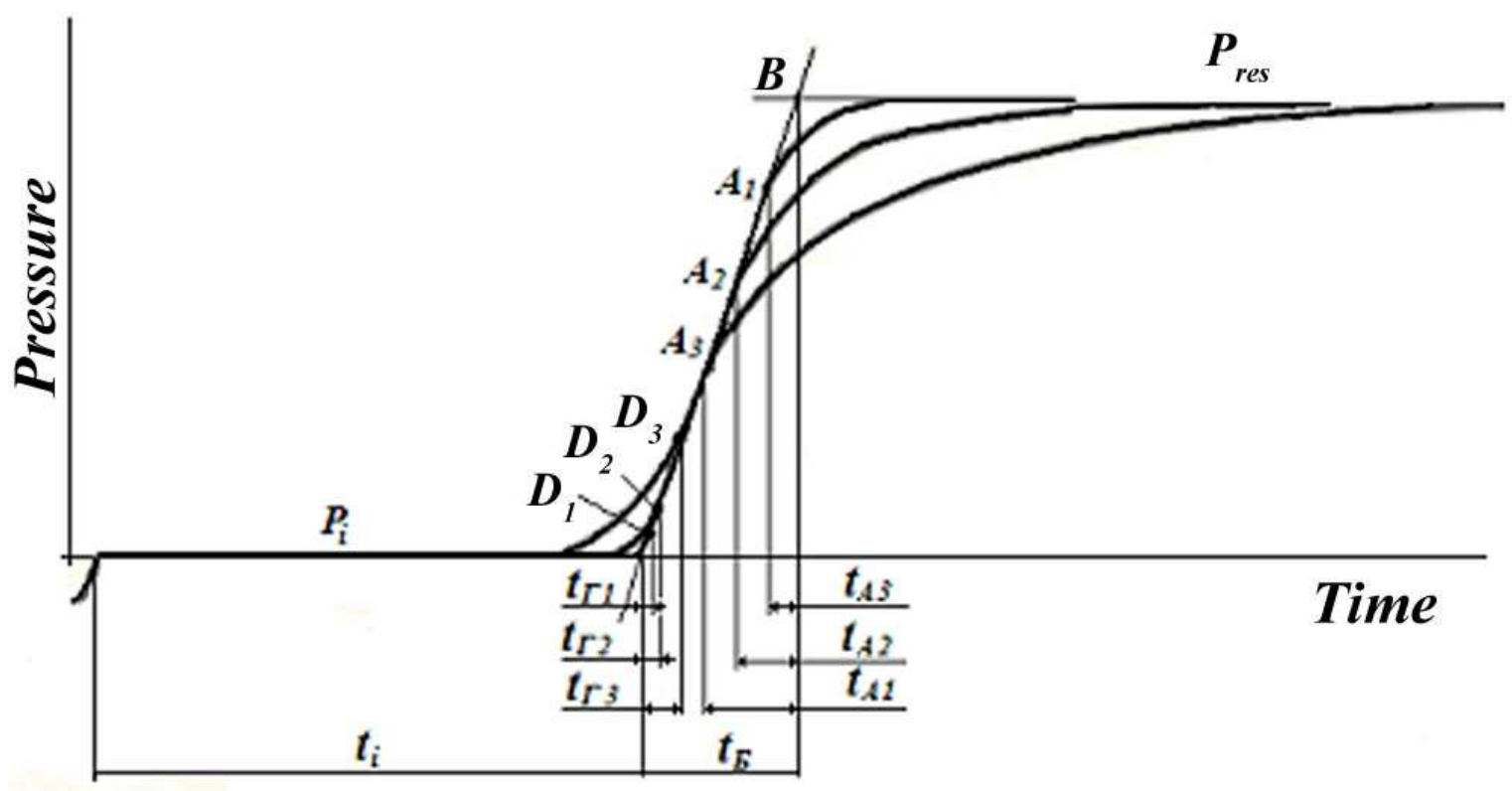

Fig. 1. Amplitude and time elements of transient process of pressure diagram

Despite the appreciable scatter of $t_{B}\left(t_{i}\right)$, one can note a trend of points to group according to the type of medium: gas, water or oil. For instance, for gas gas-bearing zones, the dependence $t_{B}\left(t_{i}\right)$ is characterized by more time of elastic compression $t_{B}$ in relation to the time of inflow $t_{i}$ which confirms the hypothetical view of the diagrams of gas-bearing zones in Fig. 1. For water-bearing zones, the dependence $t_{B}\left(t_{i}\right)$ is characterized, as a rule, by the region of small inflow time, i.e. high phase permeability for water (filtrate) and lesser angle of $t_{B}$ to $t_{i}$; for oil-bearing zones, large inflow time $t_{i}$ and intermediate-between gas and water-values of $t_{B}$ are typical.

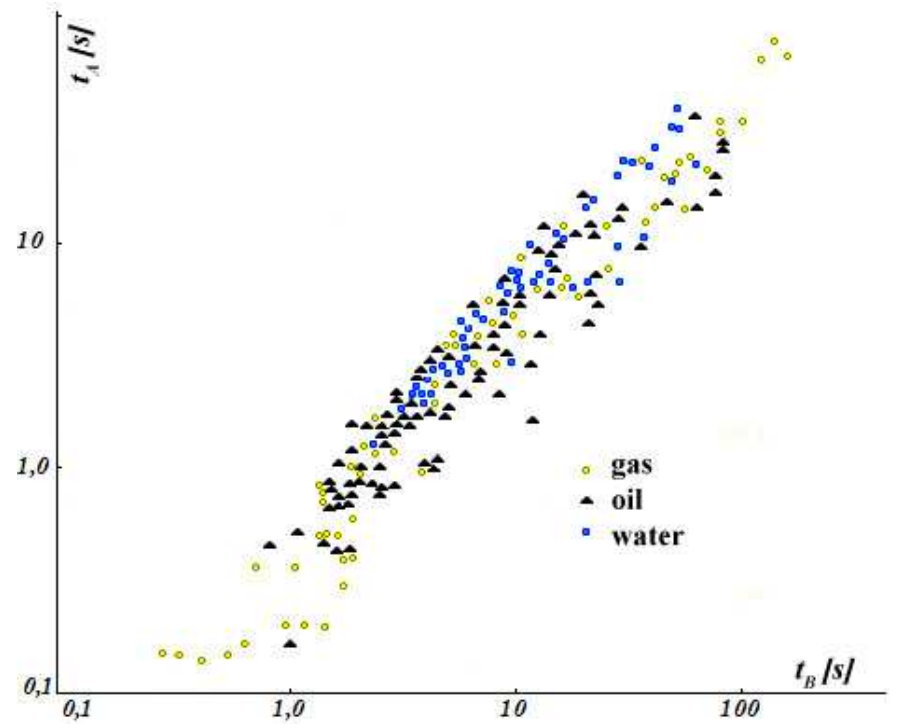

Fig. 2. Time of leaving the slope $t_{A}$ vs. transient process time $t_{B}$

The scatter of $t_{B}\left(t_{i}\right)$ can be explained by the influence of clogging zone, insufficient bearing-out of clay particles from near-well bore area in the vicinity of the inflow that got there 
during filtration of the flushing liquid, and formation of a wall cake. The influence of clogging expressly increases $t_{i}$ and is not significant during elastic period $t_{B}$. This is confirmed by the plot of the time of curve leaving the section of maximum inclination $t_{A}$ and time of transient process $t_{B}$ (Fig. 2). Here the dependence of $t_{B}\left(t_{i}\right)$ is more determined and for upper boundary values can be expressed by the following empirical equation:

$$
t_{B}=k+t_{A}^{n},
$$

where $n$ is curve inclination; $k$ is the section from point of reference to the intersection of the curve $t_{A}$ with axis $t_{B}$.

The values of $t_{B}$ and $t_{A}$ are classified according to the saturation character of areas studied during hydrodynamic survey. Relative deviation of $t_{B}$ and $t_{A}$ in different zone areas characterizes the change to the elastic properties of compressed multi-component fluid, which is conditioned by different content of components in bottom-hole area, including residual ones.

Indeed, when distinguishing the transient process phases during processing of pressure diagram, in particular, time of leave $t_{A}$ for different fluids, it was noted that for "pure" fluids (water and oil), time $t_{A}$ significantly differs. At the same duration of transient process $t_{B}$, small time $t_{A}$ is typical for water (filtrate), while large $t_{A}$ is typical for oil, i.e. the leave of the slope for it is earlier. Physically it can be easily explained by large coefficient of oil compression as compared to water. This difference can be increased by the fact that the factor which lowers time $t_{B}$ for water is large TCLE of water. The compression is adiabatic, especially at fast inflows, and the rate of pressure rise at the end stage of transient process is either constant, or can be quite large, which causes a sharp inflection of the curve $P_{\text {res }}$ turning into asymptote.

For purely gas objects, as a rule having small flushed zone (at least, for our examples), $t_{A}$ is small, as compared to the transient process time, which is explained by linear gas compression law and stability of the phase composition. Time $t_{D}$ was determined from pressure diagrams recorded by AGIP$\mathrm{K}$ during the fluid inflow into the void, i.e. at maximum drawdown.

To estimate the saturation of the reservoir area studied by hydrodynamic survey, the section being complicated by a flushed zone and containing both filtrate and product, one can assess the fraction of one or another component by the difference in coefficients of compression and its effect on the amplitude-time characteristic of the transient process, expressed for instance as indicator $\beta=t_{A} / t_{B}$. In addition, a particular interest is in a comparative study of filtration and elastic components of the process which can be expressed as indicator $\alpha=t_{B} / t_{i}$. In some cases, one may use indicator $\gamma=t_{D} / t_{B}$ which characterizes residual gas saturation of the zone. Fig. 3 illustrates the statistical data as distribution plots for the three indicators for typical objects.

Indicator (filtration-elastic characteristic) $\alpha$ in gas-bearing zones of Yakutia in $40 \%$ of all cases $\alpha>0,8$; in the rest of cases they are quite uniformly distributed from 0.1 up to 0.7 .
In oil-bearing zones, the major volume is occupied by $\alpha=0.1 \ldots 0.3$ (86\%), including $\alpha=0 \ldots 0.1$ (37\%), $\alpha=0.1 \ldots 0.2$ $(33 \%)$. In water-bearing, watercut and transition zones of OWC, in $70 \%$ of cases $\alpha=0 \ldots 0.1$ and is limited by $\alpha<0.3$.

Thus, in terms of indicator $\alpha$, the saturation by gas is confidently identified in gas-bearing zones. Regarding oil- and water-bearing zones, oil content can be identified only in $15 \%$ of cases for the indicator ranging from 0.3 to 0.9 .

Presence of common region $\alpha$ (oil) and $\alpha$ (water) is explained by primary saturation of the studied zone by flush liquid filtrate.

Indicator $\beta$ (elastic-capacitive characteristic) is less identifying for the saturation estimation by elastic indicators, because the saturation indicators for pressure build-up of water, oil and gas are blurred on the whole range. For the majority of cases for all types of fluids, indicator $\beta=0.5 \ldots 0.6$. This indicator is very sensitive to any deviations of the elastic characteristic of transient process, caused by the presence of residual oil or small quantities of dissolved gas. That is why the uniformity of $\beta$ distribution, probably, can be explained by the presence of oil additives or other components of the flush liquid in the studied zone.

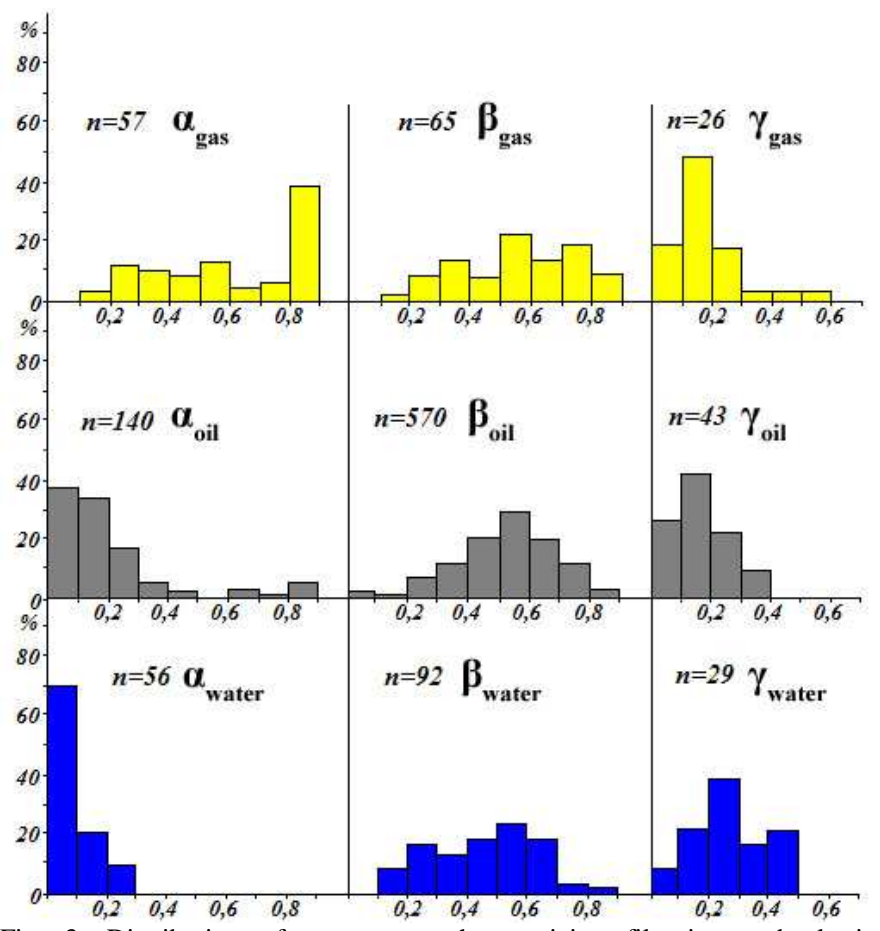

Fig. 3. Distribution of parameters characterizing filtration and elastic properties of rocks and fluids of gas and oil bearing zones (Yakutia, Tatarstan, Western Siberia).

Indicator $\gamma$ (gas content) for the maximum number of cases in gas zones is $0.1 \ldots 0.2(50 \%), 0 \ldots 0.1(25 \%)$ and $0.2 \ldots 0.3$ (20\%); the rest are uniformly distributed as $4 \%$ from 0.3 to 0.6 . In oil-bearing zones, maximum $\gamma=0.1 \ldots 0.2$ (42\%), from 0 to $0.1(26 \%)$ and from 0.2 to $0.4(32 \%)$. In water-bearing zones, maximum $\gamma=0.2 \ldots 0.3$ (39\%); the rest values up to 0.5 constitute $61 \%$ with quite uniform distribution. 


\section{CONCLUSIONS}

Hydrodynamic survey technology, along with rapid determination of the reservoir pressure, during detailed analysis of transient processes under pressure build-up, allows qualitatively estimating filtration-elastic characteristics, elastic-capacitive characteristics and gas content of pay zones. The systematic filling of databases with data from studied zones may allow performing quantitative assessment of these indicators.

\section{References}

[1] Cars. Ah, N. Randy, I. Jonah, Computerization of the machine hiphydrodynamic Carthage and testing, TV "basket", Tver: Ed. As, Vol.93, pp. $125-128,2002$.

[2] A. A. Car, P. Stocks, E.M. Mark, M.R. Bashar, Development of hydrodynamic aperture Carthage and test posts hip-K, TV "basket", Tver, Vol. 5 (203), pp. 202-208, 2011.

[3] A. A. Machine, Definition posts pressure hip To the diaphragm technology high-cycle Sandra, TV "basket", Tver: Ed. How, Vol. 3 (273), pp. 62-66, 2017.

[4] A.A. Machine, I. Ion, M. Cos, C. Kashyk, Hydrodynamic cottage with electric Sodium perturbation zone, Geology of oil and gas, No. 2, pp. 2224, 1993.

[5] A. A. Machines, I. Ion, Possibility of the study of reservoir rocks is complex Aperture GDK-MBMK, TV "basket", Tver: Ed. As, Vol.1 (114), pp. 152-157, 2004.
[6] N. Randy, I. Relax, L.E. Keller, From The Trail.Mmm, Hydrodynamic basket in open stole sin using domestic diaphragm on cable (latest results and scores), TV "basket". Tver: Ed. As, Vol. 203, pp. 191-201, 2011.

[7] N. Randy, E.M. Mark, V. Sugar, N.A. Nikolaev, M.R. Bashar, Test of posts and selection globe Pro Aperture for cable, TV "basket", Tver: Ed. As, Vol. 7-8 (148-149), pp. 255-272, 2006.

[8] X RAM, Review of hydrodynamic studies sin in open and cased stole module static place on cable MDT / CHDT, Neftegaz obozrenie, pp. 3045, 2004.

[9] K Jan. et al. New aspects of the posts instrument test on cable, Neftegaz review, 1, pp. 96-108.

[10] V. E. B. Dussan Y. Sharma, Analysis of pressure Single-probe formation tester. SPE 160801, 1992.

[11] CR H. Ashurov, An overview of hydrodynamic studies of sin in open and cased stole the module Staten place MDT/CHDT, Neftegaz display, pp. 30-45, 2005.

[12] E. S. Mathews D. Russell, Build-up of pressure and flow rates in wells, Monograph, Society, a number of Petroleum engineers in Dallas me, 1, Chap. 2, 1967.

[13] S. Ramey Gringarten Alam, Henry George. Jr., R. Raghavan, Pressure Analysis for Fractured wells, paper SPE 4051 presented at SPE AIME 47th annual fall meeting, San Antonio, Texas., October. 8-11, 1972.

[14] R. C. Earlougher, Jr. Estimating Drainage Shapes From The Limited Tests Of The Tank, George. Pet. Technology (Oct. 1971), pp. 1266-1268, Trans. AIME, 251. 